\title{
SUB-HARMONIC AND RATIONAL SYNCHRONIZATION FOR PHASE-NOISE IMPROVEMENT
}

\author{
S. Ver Hoeye, L. Gutiérrez, S. Sancho, A. Suárez, P. González \\ Dtpo. Ingeniería de Comunicaciones. University of Cantabria, Avda. Los Castros s/n, \\ 39005 Santander, Spain, almu@dicom.unican.es
}

\begin{abstract}
In an injected oscillator, synchronization bands (or Arnold tongues) are theoretically obtained at rational ratios between the frequencies of the generator and the self-oscillation. Here an in-depth analysis of this phenomenon is carried out. A simple and accurate technique is presented for the harmonic-balance prediction of the subharmonic and rational synchronization bands. Two designs of a synchronized MESFET-based oscillator at the respective ratios $1 / 4$ and a $3 / 4$ are presented, with the simulation and measurement of the steady-state regime. A good improvement of the freerunning oscillator phase noise has been achieved.
\end{abstract}

\section{INTRODUCTION}

The connection of an external generator (at a frequency $\omega_{\text {in }}$ ) to a free-running oscillator (at the frequency $\omega_{a}$ ) generally gives rise to a quasi-periodic regime, at the two fundamental frequencies $\omega_{\text {in }}$ and $\omega_{\mathrm{a}}$, with $\omega_{\mathrm{a}}$ being the self-oscillation frequency, slightly modified under the influence of the injection generator. When representing the resulting steady-state regime in the phase space, a torus is obtained, with the solution filling the torus surface [1]. A ratio can be defined between the two fundamentals, called rotation number: $\mathrm{r}=\frac{\omega_{\text {in }}}{\omega_{\mathrm{a}}}$.

When a parameter, such as the input-generator amplitude or frequency, is modified, $r$ evolves and for rational values, $r=\mathrm{m} / \mathrm{n}$, an entrainment phenomenon takes place, giving rise to a single closed trajectory in the torus, attracting all the former trajectories. Two saddle-node bifurcations determine the end of synchronized behavior [1]. Locking becomes increasingly likely as the forcing amplitude is increased, the region of synchronized solutions forming what is called an Arnold tongue. On the other hand, the length of the synchronization intervals decreases rapidly with $m$ and $n$ [2], which explains why most of the Arnold tongues are not experimentally observed.

This work was motivated by the need to reduce the number of synthesized oscillators in a transmitter-receiver system, by using the output of one synthesizer at $\omega_{1}$ to synchronize a VCO at $\omega_{2}$ $=\mathrm{m} / \mathrm{n} \omega_{1}$. The objective has been the investigation of the stability and synchronization properties of Arnold tongues $\mathrm{m} / \mathrm{n}$ [1]. An example of this kind of operation can be found in phase-locked loops, based on sampling phase-detectors [3]. In these systems, phase locking is obtained at a high-order harmonic component of the crystal-oscillator frequency and thus, at an Arnold tongue $1 / \mathrm{k}$. The synchronization bands are very narrow and the VCO has to be tuned to achieve phase locking. Similar behavior is expected here.

\section{CIRCUIT DESIGN}

Due to the expected narrowness of the $\mathrm{m} / \mathrm{n}$ synchronization bands, the free-running oscillator must be a tunable one. A MESFET-based voltage-controlled oscillator at $12 \mathrm{GHz}$ has been designed here. The oscillation condition is fulfilled at the drain port through source feedback (including a varactor 
diode). An output filter, at $\omega_{\mathrm{a}}$ is also used, with rejection of the input frequency. Two different designs have been carried out, one of them for sub-harmonic synchronization at $\omega_{\text {in }}=\omega_{a} / 4$ and the other for ultra-subharmonic synchronization at $\omega_{i n}=3 / 5 \omega_{\mathrm{a}}$. In both cases, the transistor bias was selected to improve the multiplying capabilities [2] of the circuit with respect to $\omega_{\text {in }}$. These are evaluated through mixing analysis (at the two fundamental frequencies $\omega_{\text {in }}$ and $\omega_{\mathrm{a}}$ ), with the aid of an auxiliary generator at the self-oscillation frequency [4].

\section{GRAPHICAL PREDICTION OF SYNCHRONIZATION BANDS THROUGH PHASE DIAGRAMS}

The subharmonic $(1 / \mathrm{n})$ and ultra-subharmonic $(\mathrm{m} / \mathrm{n})$ synchronization bands are very critical and generally difficult to predict. Approximate expressions, requiring measurements of output power, were provided in [2]. Here a technique based on the tracing of phase diagrams has been employed. A phase diagram is the polar representation of an impedance or admittance function using the phase as an implicit parameter. The synchronization band at $\mathrm{m} / \mathrm{n}(\mathrm{m}=1$ or $\mathrm{m} \neq 1)$ is analyzed through harmonic balance, using a Fourier-series expansion at the fundamental frequency $\omega_{\mathrm{f}}=\omega_{\mathrm{a}} / \mathrm{n}$. Thus the oscillation frequency is $\omega_{\mathrm{a}}=\mathrm{n} \omega_{\mathrm{f}}$ and the input generator operates at $\omega_{\mathrm{in}}=\mathrm{m} \omega_{\mathrm{f}}$. The use of an auxiliary generator (AG) at the oscillation frequency $n \omega_{\mathrm{f}}$ enables tracing the phase diagrams. The AG (only introduced for simulation purposes) may be a voltage generator, connected in parallel at a circuit node, or a current generator, in a series connection. The AG frequency is the same as the oscillation frequency $\omega_{\mathrm{a}}=\mathrm{n} \omega_{\mathrm{f}}$ and an ideal filter is also necessary [4] to prevent the influence of the $\mathrm{AG}$ at frequencies different from $\omega_{\mathrm{a}}$.

To obtain the phase diagrams, a double sweep is carried out in the AG amplitude and phase, determining the corresponding input impedance (for a current $A G$ ) or admittance (for a voltage AG). When representing this magnitude in a polar plot, due to the phase periodicity, a closed curve is obtained for each AG amplitude. The synchronized solutions are given by the intersections with the plot origin. When also varying the input generator frequency, families of curves are obtained. The synchronization band (between $m \omega_{\mathrm{f} 1}$ and $m \omega_{\mathrm{f} 2}$ ) is given by the tangential intersections of the families (respectively at $m \omega_{\mathrm{f} 1}$ and $\mathrm{m} \omega_{\mathrm{f} 2}$ ) with the plot origin. In the subharmonic design, a bandwidth of $5 \mathrm{MHz}$ was obtained, for input power $3 \mathrm{dBm}$ at $3 \mathrm{GHz}$. The design for $\mathrm{m} / \mathrm{n}=3 / 5$, with $7 \mathrm{GHz}$ input frequency, provides $2 \mathrm{MHz}$ for the same input power (Fig. 2). Larger synchronization bands are obtained for higher input power.

\section{STEADY-STATE SYNCHRONIZED SOLUTION}

For obtaining the steady-state synchronized solution at $\mathrm{m} / \mathrm{n}$, optimization of the auxiliary generator is carried out. In these optimization, (and for each varactor bias), the variables are the amplitude, phase and frequency $\omega_{\mathrm{f}}$. The resulting solution in time domain is represented in Fig. 2, together with the 2-torus prior to synchronization. The variables employed in the representation are determined by

the $v_{G S}$ and $v_{D S}$ and the phase of the ultra-subharmonic $\theta=\frac{2}{3} \omega_{i n} t$. The system solution is on the surface of the torus, the longitudinal angle being related to the external frequency and the latitudinal angle to the natural frequency. In the quasi-periodic state the orbit fills the torus surface. When the system approaches the synchronized state, certain region on the torus becomes more attractive than the rest of the surface, and the orbit spends more time around it. In the synchronized state, both frequencies are harmonically related, and there is a closed orbit that attracts all the trajectories on the torus. This closed orbit lies on the former attractive region. When the system is synchronized, the fundamental frequency is $\omega_{\mathrm{f}}=\omega_{\mathrm{a}} / 5=\omega_{\mathrm{in}} / 3$, and the orbit is closed over itself after two periods of $\theta$. This corresponds to five periods of the autonomous component at $\omega_{\mathrm{a}}$. 
A Nyquist stability analysis [5] of this solution has also been carried out, obtaining the stable plot of Fig. 3. The solution is stable, although the stability margin appears to be small, due to the reduced synchronization bandwidth.

\section{COMPARISON OF SIMULATED AND EXPERIMENTAL RESULTS}

The synchronized circuit was manufactured and experimentally characterized. Phase locking was achieved from $1 \mathrm{dBm}$ input power. Fig. 4 shows the spectrum of the simulated synchronized solution, with the experimental points superimposed. Fig. 5 shows experimental comparison between the output spectrum of the free-running and the phase-locked oscillator, in a reduced span of $3 \mathrm{MHz}$, for a bias voltage with higher output power. The circuit did not unlock in long term measurements of two days and was stable in the temperature range $0-50{ }^{\circ} \mathrm{C}$.

\section{CONCLUSIONS}

A theoretical study of synchronization at subharmonic and ultra-subharmonic orders, for possible phase-noise improvement applications, has been carried out here. A technique has been provided for determining the narrow synchronization bands. Two $12 \mathrm{GHz}$ MESFET-based oscillators have been designed, to, respectively, operate in the synchronization bands $1 / 4$ and 3/5. Actual synchronization was experimentally obtained in both cases, with good reduction of phase noise. However, the freerunning oscillator must be tunable to initially achieve the lock. To our knowledge it is the first time that ultra-subharmonic synchronization has been simulated in a practical oscillator and experimentally obtained.

\section{ACKNOWLEDGEMENT}

This work was supported by the FEDER project 1FD97-0455-C02-01 (TIC).

\section{REFERENCES}

[1] J. Guckenheimer, P. Holmes; "Nonlinear oscillations, dynamical systems and bifurcations of vector fields"; New York,1990; Springer-Verlag: Third printing.

[2] X. Zhang, X. Zhou, B. Aliener, A. Daryoush; "A study of subharmonic injection for local oscillators"; Mar. 1992; IEEE Microwave and Guided Wave Letters: vol. 2, no. 9.

[3] S. Sancho, S. Ver Hoeye, A. Suárez, J. Chuan, A. Tazón; "Nonlinear analysis of a microwave synthesizer, based on a sampling phase detector"; May, 2001; IEEE MTT-S Phoenix (USA).

[4] V.Iglesias, A.Suárez, J.L.García; "New technique for the determination through commercial software of the stable-operation parameter ranges in nonlinear microwave circuits"; Dec. 1998; IEEE Microwave and Guided Wave Letters: Vol. $8 \mathrm{~N}^{\circ} 12$; 424-426.

[5] A. Suárez, V. Iglesias, J. M. Collantes, J. Jugo, J. L. García; "Nonlinear stability analysis of microwave circuits using commercial software"; Jun. 1998; IEE Electronic Letters: vol. 34, no. 13; pp. 1333-1334. 


\section{FIGURES}

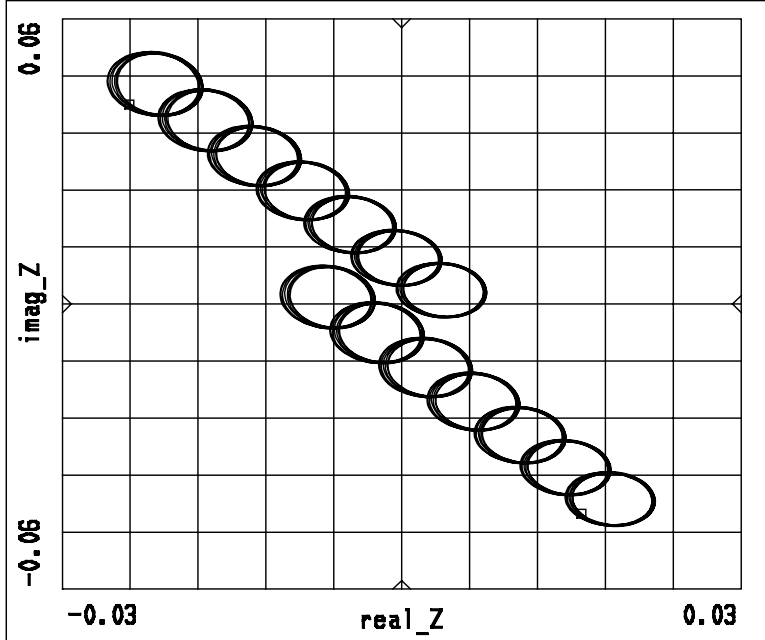

Fig. 1 Graphic determination of synchronization bands

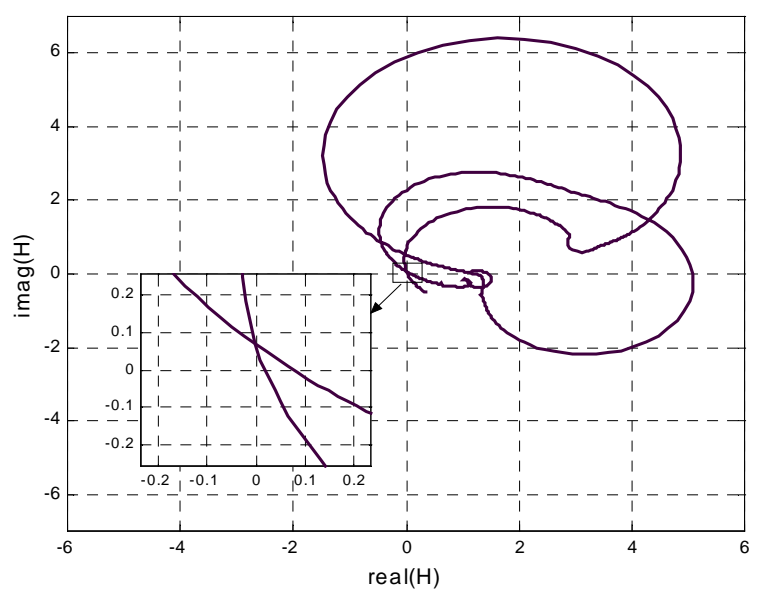

Fig. 3 Nyquist stability plot for the $\mathrm{m} / \mathrm{n}$ entrained solution.

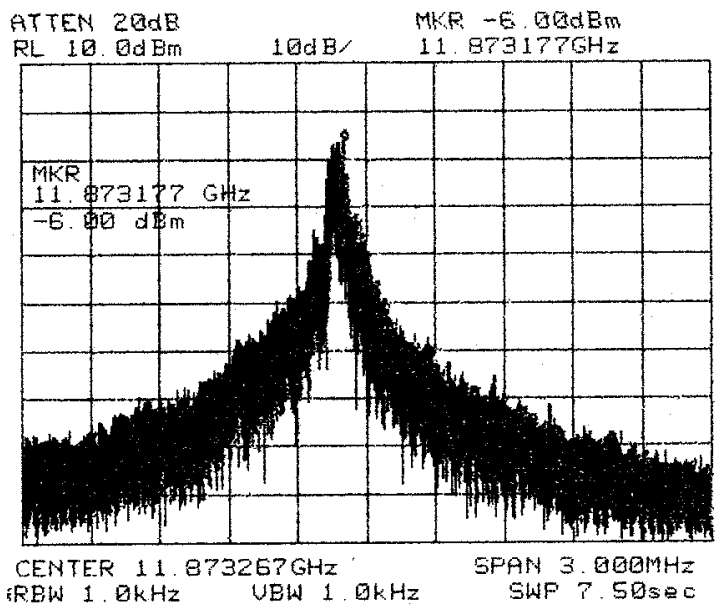

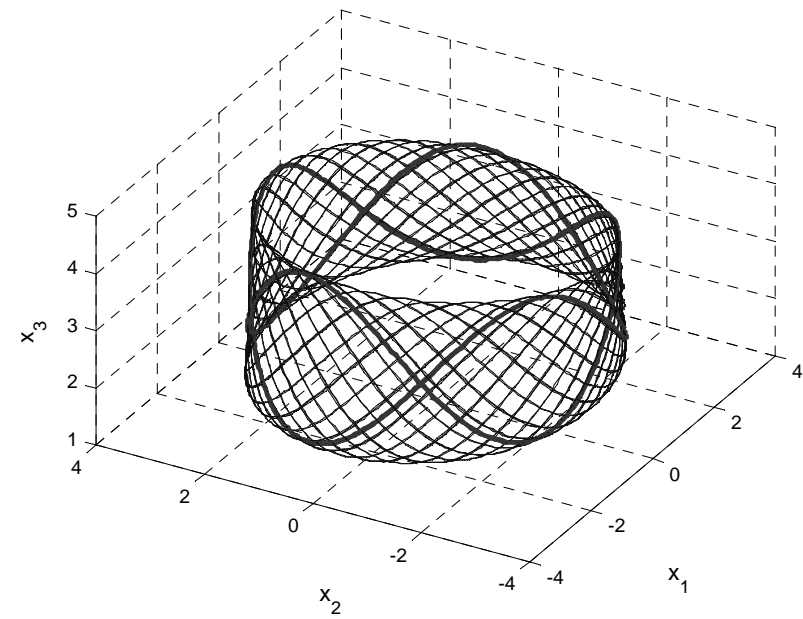

Fig. 2 3/5-Entrained solution in the 2-torus, for the MESFET-based oscillator circuit.

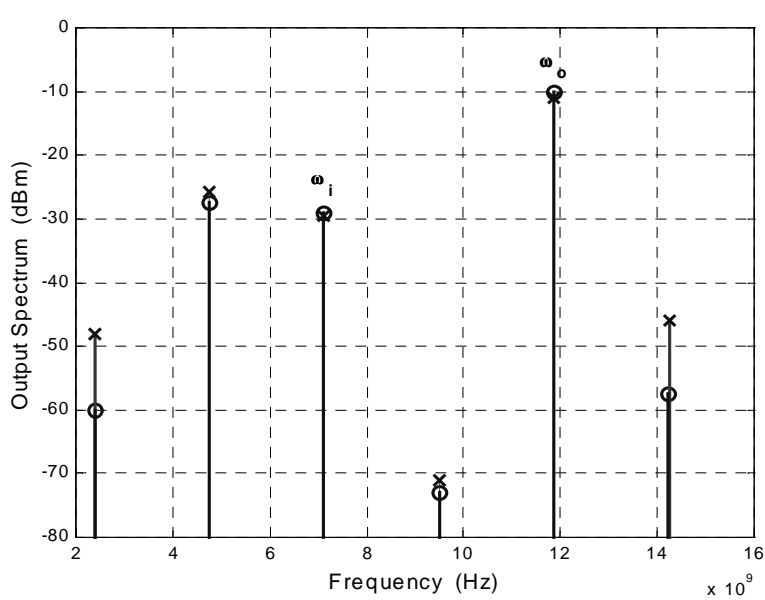

Fig. 4 Spectrum of $3 / 5$ synchronized solution with experimental points superimposed

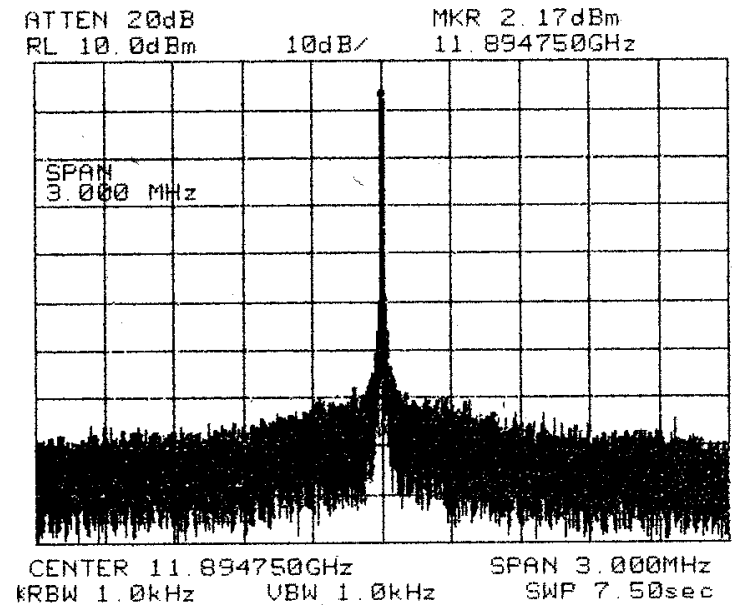

Fig. 5 Measurement of the output spectrum of the $12 \mathrm{GHz}$ oscillator. (a) Free-running behavior. (b) Synchronized behavior, using a $7.137 \mathrm{GHz}$ input generator. 